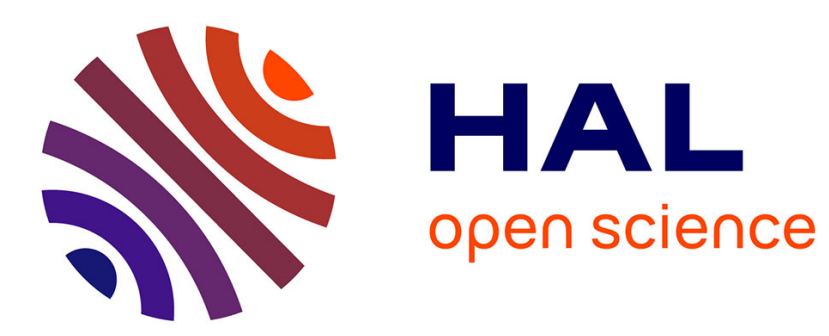

\title{
Dealing with Multi-Policy Security in Large Open Distributed Systems
}

\author{
Christophe Bidan, Valérie Issarny
}

\section{To cite this version:}

Christophe Bidan, Valérie Issarny. Dealing with Multi-Policy Security in Large Open Distributed Systems. [Research Report] RR-3112, INRIA. 1997. inria-00073578

\section{HAL Id: inria-00073578 \\ https://hal.inria.fr/inria-00073578}

Submitted on 24 May 2006

HAL is a multi-disciplinary open access archive for the deposit and dissemination of scientific research documents, whether they are published or not. The documents may come from teaching and research institutions in France or abroad, or from public or private research centers.
L'archive ouverte pluridisciplinaire HAL, est destinée au dépôt et à la diffusion de documents scientifiques de niveau recherche, publiés ou non, émanant des établissements d'enseignement et de recherche français ou étrangers, des laboratoires publics ou privés. 
INSTITUT NATIONAL DE RECHERCHE EN INFORMATIQUE ET EN AUTOMATIQUE

\section{Dealing with Multi-Policy Security in Large Open Distributed Systems}

Christophe Bidan \& Valérie Issarny

\section{$\mathbf{N}^{\circ} 3112$}

Février 1997

THÈME 1

apport

derecherche 



\title{
RINRIA
}

\section{Dealing with Multi-Policy Security in Large Open Distributed Systems}

\author{
Christophe Bidan \& Valérie Issarny \\ Thème 1 - Réseaux et systèmes \\ Projet Solidor \\ Rapport de recherche $\mathrm{n}^{\circ} 3112$ - Février $1997-20$ pages
}

\begin{abstract}
From the security point of view, one challenge for today's distributed architectures is to support interoperation between applications relying on different possibly inconsistent security policies.

This paper proposes a practical approach for dealing with the coexistence of different security policies in distributed architectures. We introduce a model for specifying security policies in terms of security domains and access control rules. Then, we identify the set of operators for combining the specifications of sub-policies and we address the validity of the resulting policy according to the security properties of the sub-policies.
\end{abstract}

Key-words: Access control policy, information flow policy, multi-policies, conflicting policies. 


\section{Gestion de multiples politiques de sécurité dans un système distribué ouvert}

Résumé : Du point de vue de la sécurité informatique, les architectures distribuées actuelles doivent pouvoir supporter l'interopération entre applications relevant de politiques de sécurité différentes, éventuellement contradictoires.

Ce papier propose une approche pratique pour traiter la coexistence de différentes politiques de sécurité dans une architecture distribuée. Nous introduisons un modèle permettant de spécifier les politiques de sécurité en termes de domaines de sécurité et de règles de contrôle d'accès. Nous identifions alors un ensemble d'opérateurs pour combiner les spécifications des sous-politiques, et nous nous intéressons à la validité de la politique résultante vis-à-vis des propriétés de sécurité des sous-politiques.

Mots-clé : Politique de contrôle d'accès, politique de contrôle de flux, multi-politiques, conflit entre politiques. 


\section{Introduction}

Object-based distributed computing architectures ${ }^{1}$ like CORBA (Common Object Request Broker Architecture) defined by the Object Management Group (OMG) [27], and the Telecommunication Intelligent Network Architecture proposed by the TINA consortium [30] are promising approaches to support large open distributed systems. Goals of these architectures include interoperability between software components in heterogeneous distributed environments, where components may appear and disappear dynamically, as the result of individual and autonomous actions. From the security point of view, the interoperability promoted by the above architectures stresses the complexity of security policies which have to be implemented $[15,10]$, as well as the necessity to cope with the coexistence of multiple security policies. This coexistence results from the interoperation between systems (or software components) having different security requirements, possibly at different granularity levels.

For evaluating the security of open distributed systems, security officers should be able to reason about the composition of the interoperating systems' security policies. We identify two different approaches for the composition of security policies: policy interoperation and policy combination. Using policy interoperation, the composed policy should not violate the security of the sub-policies, and should guarantee their autonomy [14]. On the other hand, with policy combination, the composed policy may be inconsistent with both subpolicies, but must be secure in the given context. Hence, security combination allows to compose conflicting policies in a secure and controlled manner [3]. In the context of large open distributed architectures, we assert that policy combination is better suited than policy interoperation. In this paper, we introduce a model that forms the basis for dealing with security in heterogeneous distributed architectures, allowing to specify and to combine a wide variety of access control policies. In particular, the proposed model is useful to security officers by supplying a practical set of combination operators.

This paper is structured as follows: the next section presents related work and gives our standpoint concerning policy composition. Section 3 presents our model for the specification of strict access control policies (without considering information flow). In particular, it gives the notion of complete and sound combination. Section 4 extends this model so as to deal with combination of information flow policies. Finally, we draw some conclusions in section 5 and present current status and future work.

\section{Topics for Policy Combination}

Various models have been proposed in order to reason about security policies (e.g., see an overview of security models by Landwehr [20], the Bell-LaPadula model [4], the information flow model from Bieber [7], the Clark-Wilson model for commercial security constraints [9], and the McLean model [23]). These models are introduced to check whether a policy

\footnotetext{
${ }^{1}$ From the security point of view, an object-based distributed computing architecture is viewed as multiple interconnected systems [26].
}

$\mathrm{RR} \mathrm{n}^{\circ} 3112$ 
verifies given security properties like the nondeducibility property [29] or the noninterference property [13]. However, these models do not deal with the composition of policies.

Concerning the definition of a security policy resulting from the composition of policies, we identify two different approaches:

- policy interoperation which infers the composed policy based on the security properties of the sub-policies, and

- policy combination which specifies the composed policy based on the specifications of the sub-policies.

The former approach relies on the principles of autonomy and of security of sub-policies [14]: during the interoperation of two different security policies, any access authorized within an individual policy must also be authorized within the composed policy; and dually, any access denied within an individual policy must also be denied within the composed policy. Abadi et al. [1] propose a general solution to the inference of execution properties of interoperating components from the components' specification, which can be adapted to security policies. More recently, McLean [24] has presented an approach which allows to reason about security properties of composed information flow policies. We also mention the work done by Heintze et al. [16] who propose an approach for secure protocols and focus on the problem of their composition. Finally, Gong et al. discuss computational issues concerning the general secure interoperation problem [14].

The above proposals allow to verify that interoperation preserves the sub-policies' security properties under certain conditions. However, the undertaken approach to the definition of policy composition is restrictive; the security properties of the composed policy must be the same as those of the sub-policies (the principles of autonomy and security must be guaranteed). In other words, sub-policies have to be compatible. In large open distributed system, this is not always the case, since the properties of a policy resulting from the composition of two incompatible sub-policies can be an extension or a restriction of those sub-policies. We illustrate this through two examples.

In both examples, we view the system as a collection of entities (i.e. software components). We say that an entity $\epsilon_{1}$ is authorized to access the entity $\epsilon_{2}$ if and only if $\epsilon_{1}$ is authorized to access $\epsilon_{2}$ 's data. We specify only authorized accesses, and all accesses that are not specifically authorized, are denied (except for the reflexive accesses: each entity is authorized to access itself).

Let us first consider a file system where we have two sets of users, $A$ and $C$, and two sets of files, $B$ and $D$. We define the access control policy $\mathcal{P}_{1}$ (resp. $\mathcal{P}_{2}$ ) managing $A$ users and $B$ files (resp. $C$ users and $D$ files): $\mathcal{P}_{1}$ (resp. $\mathcal{P}_{2}$ ) authorizes users in $A$ (resp. in $C$ ) to access files in $B$ (resp. in $D$ ). Let us now combine $\mathcal{P}_{1}$ with $\mathcal{P}_{2}$ to build the following composed policy: the users belonging to both sets $A$ and $C$ (denoted as $A \cap C$ ) are authorized to access the files in $B \cap D$, users in $A$ but not in $C$ (denoted as $A-C$ ) are authorized to access the files in $B-D$, and finally, users in $C-A$ are authorized to access the files in $D-B$. 
As a second example, we consider a military system consisting of a set of users and files that the users manipulate. Suppose that we have two classifications for both files and users: classification in terms of security level (i.e. Secret and Unclassified), and classification in terms of military domains (i.e. Nuclear and Conventional). Let $A, B, C$ and $D$ be the sets of Secret, Unclassified, Nuclear and Conventional entities, respectively. Then, regarding read access, we specify policy $\mathcal{P}_{1}$ to authorize $A$ entities to access $B$ entities, and policy $\mathcal{P}_{2}$ to authorize $C$ entities to access $D$ entities. One way to compose these sub-policies gives the following composed policy: $C$ entities with $A$ classification are authorized to access $D$ entities with $A$ classification, which in turn are authorized to access $C$ entities with $B$ classification, these latter being authorized to access $D$ entities with $B$ classification.

The composed policy of the first example is a restriction of the participating sub-policies, whereas the composed policy in the second example is an extension of the participating sub-policies (Conventional entities are authorized to access Nuclear entities with different security classifications). These two composed policies are secure in the context in which they are specified, nevertheless they cannot be obtained by policy interoperation.

The specification of a policy based on the specifications of its sub-policies allows to combine possibly inconsistent policies. In particular, a combined policy does not necessarily guarantee all the properties of the sub-policies. Hosmer [17] has introduced the notion of metapolicies, or "policies about policies", an informal framework for combining security policies. McLean [22] seems to be the first to propose a formal approach including combination operators: he introduces an algebra of security which enables to reason about the problem of policy conflict $^{2}$. However, even though this approach permits to detect conflicts between policies, it does not propose a method to resolve the conflicts and to construct a security policy from inconsistent sub-policies. Following Hosmer's work, Bell formalizes the combination of two sub-policies with a function (policy combiner), and introduces the notion of policy attenuation to allow the composition of conflicting security policies [3].

Our work is placed in the framework of (access control) policy combination, and it is close to Bell's approach. More specifically, our model is a practical alternative solution to Bell's proposal: we clearly identify the set of combination operators enabling security officers to combine access control policies in a controlled and secure way. Briefly, our model allows to specify the general behavior of sub-policies. Based on these specifications, we introduce combination operators for combining policy specifications. Finally, we verify that the behavior of the composed policy is secure and conforms with sub-policies specifications.

In this paper, access control policies are considered as a subset of information flow policies (or flow policies). An access control policy is defined as a security policy that only checks the accesses between entities, without verifying the information flow resulting from access operations. The following sections introduce our model to deal with access control policies and flow policies. We show how this model allows to specify such policies, and to combine their specification in a secure way.

\footnotetext{
${ }^{2}$ Close to this framework, notice the more recent work of $[19,18]$.
} 


\section{Specifying Access Control Policies}

In this section, we concentrate on the following type of access control: is an entity $\epsilon_{1}$ authorized to access an entity $\epsilon_{2}$ in the context of a given policy, regardless of the information flow that is implied by this access?

Our approach to policy composition relies on the definition of a set of combination operators. A policy is specified either as an elementary policy or a combined policy built from existing sub-policies through the use of combination operators. The next sub-section addresses the specification of access control policies and is followed by the definition of the operators allowing to combine policy specifications. Finally, we address the completeness and soundness of policy specifications.

\subsection{Elementary access control policy}

An access control policy defines a set of rules that specify for each pair of object and subject, whether the subject is allowed to access the object's state or not $^{3}$. Since in an open distributed architecture, an access control policy cannot be defined for the whole set of the system's entities, we take an approach which allows to specify the sensitive entities for each policy. In other words, an access control policy is defined not solely in terms of its access control rules but also in terms of its access control domain [25], that determines the system's objects and subjects to which the policy applies.

The access control domain of a policy is subdivided into the set of the system's entities called object domain, that contains sensitive information for this policy, and the set of the system's entities called subject domain, that gives the subjects belonging to the policy domain. These two sets are defined formally in terms of security classifications ${ }^{4}$. Given a security property $P$ (i.e. a predicate) defined on the set $\mathcal{E}$ of the system's entities, the set of entities verifying this property is termed the $P$ classification and is noted $C l_{P}$ :

$$
C l_{P}=\{\varepsilon \in \mathcal{E} \mid P(\varepsilon) \equiv \text { true }\}=\{\varepsilon \in \mathcal{E} \mid P\}
$$

Given the definition of classification, an access control domain is defined as a set of classifications:

$$
D o m=\left\{C l_{i}\right\}_{i=1}^{n} .
$$

Each access control rule $\lambda$ of a given policy defines the set of subject and object couples that verify a given security property $P_{\lambda}$ (called access control predicate). Thus, a rule specifies an access control condition $C_{\lambda}$. i.e. a security property bearing on classifications of subjects and objects, and an access control operator. The access control operator is noted $\sim>$ for authorization, $\not>$ for denial, and the $\div>$ operator will be used to denote $\sim>$ or $\not>^{5}$. Formally, the access control condition takes the form $C_{\lambda}(s, o)=\left(s \in C l_{1}\right) \wedge(o \in$ $\left.C l_{2}\right) \wedge P_{\lambda}(s, o)$ where $C l_{1}$ and $C l_{2}$ are two classifications. Then, an access control rule $\lambda$ takes

\footnotetext{
${ }^{3}$ We define the subjects as active entities, and the objects as passive or active entities [11].

${ }^{4}$ We use the term classification for both object classification and subject clearance.

${ }^{5}$ We consider that each access corresponds to the execution of a given operation (e.g. read access corresponds read method). This enables us to use a single access operator.
} 


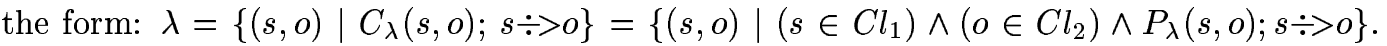
In other words, we say that a pair $(s, o)$ belongs to $\lambda$ if the access condition $C_{\lambda}(s, o)$ holds.

In the remainder, for $\mathcal{P}$ being an access control policy, its domain is noted $D o m_{\mathcal{P}}$, the notation $D o m_{\mathcal{P}}^{o}$ (resp. $D o m_{\mathcal{P}}^{s}$ ) is used to designate the sub-domain of $D o m_{\mathcal{P}}$ that defines $\mathcal{P}$ 's objects (resp. subjects). Finally, $R_{\mathcal{P}}$ is the set of access control rules of the policy $\mathcal{P}$.

\section{Example.}

We now illustrate our model for policy specification with the example of a file system expressed as a distributed architecture. Let $\mathcal{F S}$ be the set of software components accessing files. Let further $R E A D \subset \mathcal{F S}$ and $W R I T E \subset \mathcal{F S}$ be respectively the set of software components allowed to read and to write files. We also consider two sets of users, $A$ and $C$, and two sets of files, $B$ and $D$. We introduce the predicate $O w n e r(s c, U)$ which, given a software component $s c$, holds if the owner of $s c$ (i.e. the user executing $s c$ ) belongs to the set $U$ of users (i.e. $A$ or $C$ ). In the following, we do not specify reflexive accesses. Given the above definitions, we define the $\mathcal{P}_{1}$ and $\mathcal{P}_{2}$ access control policies introduced in $\S 2$, as follows:

- The subject domain of the policy $\mathcal{P}_{1}$ is the set of components $R E A D\left(D_{0} m_{1}^{s}=\right.$ $R E A D)$, and $\mathcal{P}_{1}$ 's object domain is the set of files $B\left(D o m_{1}^{o}=B\right)$. The policy $\mathcal{P}_{1}$ has a single access control rule

$$
\lambda_{1}=\{(s, o) /(s \in R E A D) \wedge(o \in B) \wedge \operatorname{Owner}(s, A) ; s \sim o\},
$$

that authorizes subjects of $A$ to access objects of $B$.

- The subject domain of the policy $\mathcal{P}_{2}$ is the set of components WRITE (Dom Do $_{2}^{s}=$ $W R I T E)$, and $\mathcal{P}_{2}$ 's object domain is the set of files $D\left(D_{o m}^{o}=D\right)$. The policy $\mathcal{P}_{2}$ has a single access control rule

$$
\lambda_{2}=\{(s, o) /(s \in W R I T E) \wedge(o \in D) \wedge \operatorname{Owner}(s, C) ; s \sim>o\},
$$

that authorizes subjects of $C$ to access objects of $D$.

\subsection{Combining access control policies}

A composed access control policy is built by combining two sub-policies. The domain and rules of the composed policy are defined in terms of the combination of its sub-policies' domains and rules. Thus, we introduce two operators for domains combination and rules combination respectively. For $\mathcal{P}_{1}=\left(\operatorname{Dom}_{1}, R_{1}\right)$ and $\mathcal{P}_{2}=\left(D o m_{2}, R_{2}\right)$ being two access control policies, and $\mathcal{P}=(D o m, R)$ being the access control policy resulting from the composition of $\mathcal{P}_{1}$ and $\mathcal{P}_{2}$, we define the operators $\mathcal{C}, \mathcal{D}$ and $\mathcal{R}$ as:

$$
\mathcal{P}=\mathcal{P}_{1} \mathcal{C} \mathcal{P}_{1}, \text { Dom }=\operatorname{Dom}_{1} \mathcal{D} \text { Dom }_{2} \text { and } R=R_{1} \mathcal{R} R_{2} .
$$

\subsubsection{Combining access control domains}

Given two classifications, four types of combination can be implemented: the union, intersection, product of classifications, and the denial of classification combination.

$\mathrm{RR} \mathrm{n}^{\circ} 3112$ 
Let $C l_{1}=\left\{\varepsilon \in \mathcal{E} \mid P_{1}\right\}$ and $C l_{2}=\left\{\varepsilon \in \mathcal{E} \mid P_{2}\right\}$ be two classifications, we formally define their union and intersection as: $C l_{1} \cup C l_{2}=\left\{\varepsilon \in \mathcal{E} \mid P_{1} \vee P_{2}\right\}$ and $C l_{1} \cap C l_{2}=\left\{\varepsilon \in \mathcal{E} \mid P_{1} \wedge P_{2}\right\}$ respectively. Concerning the product of $C l_{1}$ and $C l_{2}$ (noted $C l_{1} \uplus C l_{2}$, three separate classifications are computed: the classifications defined by the access control predicates $P_{1} \wedge \neg P_{2}, \neg P_{1} \wedge P_{2}$ and $P_{1} \wedge P_{2}$ respectively. Finally, to forbid the combination of two classifications, we introduce the operator $\chi$ defined by: $C l_{1} \chi \mathrm{Xl}_{2}=\emptyset$.

The $U$ operator allows to extend the sub-classifications. On the other hand, the classification obtained by $\cap$ operator is a restriction of the sub-classifications. Finally, the $\uplus$ operator permits to distinguish the entities belonging to a single sub-classification from the entities belonging to both sub-classifications.

The formal definition of the operator for combining domains directly follows: it amounts to specify the type of combination for each pair of classifications of the sub-policies' domains. We get the following corresponding formal definition:

Definition 1 (Combining domains) Let Dom Dom $_{1}=\left\{C l_{i}\right\}_{i=1}^{n}$ and $\operatorname{Dom}_{2}=\left\{C l_{j}\right\}_{j=1}^{m}$ be two access control domains. Let $\Delta=\left\{\delta_{i, j} \in\{\cup, \cap, \uplus, \chi \cup\}, i=1, \ldots, n, j=1, \ldots, m\right\}$ be the set specifying the combination of classifications $\mathrm{Cl}_{i}$ of $\mathrm{Dom}_{1}$ with $\mathrm{Cl}_{j}$ of Dom $\mathrm{Do}_{2}$. The combined access control domain Dom resulting from the combination of Dom with Dom $_{2}$ with respect to $\Delta$, noted $D_{o m} \mathcal{D}^{\Delta}$ Dom $_{2}$, is given by:

$$
D o m=\left\{\forall i=1, \ldots, n, \forall j=1, \ldots, m, C l_{i, j}=C l_{i} \delta_{i, j} C l_{j}\right\} .
$$

We bring to the reader's attention that a combined domain can itself be combined with another domain.

\subsubsection{Combining access control rules.}

Because both $\sim>$ and $\not>$ access operators exist, the combination of access control rules raises the problem of conflicts among rules. Thus, we distinguish between combination of non-conflicting rules and combination of conflicting rules.

Combination of non-conflicting rules. Let us first consider the combination of nonconflicting rules. Given two non-conflicting access control rules, their combination results from the combination of their access control conditions, i.e. the combination of their classifications and of their access control predicate.

Let $\lambda_{1}$ and $\lambda_{2}$ be two access control rules specifying either authorization for both or denial of access for both:

- $\lambda_{1}=\left\{(s, o) \mid\left(s \in C l_{1}\right) \wedge\left(o \in C l_{1}^{\prime}\right) \wedge P_{1}(s, o) ; s \div \circ o\right\}$ and

- $\lambda_{2}=\left\{(s, o) \mid\left(s \in C l_{2}\right) \wedge\left(o \in C l_{2}^{\prime}\right) \wedge P_{2}(s, o) ; s \div>o\right\}$.

Let us first suppose that $C l_{1}=C l_{2}$ and $C l_{1}^{\prime}=C l_{2}^{\prime}$. Then, combining $\lambda_{1}$ and $\lambda_{2}$ consists of combining the access control predicates $P_{1}$ and $P_{2}$. With respect to existing work (e.g. [2]), we identify two types of combination: the logical and $(\wedge)$ and the logical or $(\vee)$ between $P_{1}$ and $P_{2}$. Then, the $\lambda$ access control rule resulting from the combination of $\lambda_{1}$ and $\lambda_{2}$ takes one of the following forms: 
- $\lambda=\left\{(s, o) \mid\left(s \in C l_{1}\right) \wedge\left(o \in C l_{1}^{\prime}\right) \wedge\left(P_{1}(s, o) \vee P_{2}(s, o)\right) ; s \div>o\right\}$, or

- $\lambda=\left\{(s, o) \mid\left(s \in C l_{1}\right) \wedge\left(o \in C l_{1}^{\prime}\right) \wedge\left(P_{1}(s, o) \wedge P_{2}(s, o)\right) ; s \div>o\right\}$.

Informally, the logical or operator allows to combine two access control rules in such a way that the resulting access control rule preserves the authorized accesses of the sub-rules (principle of autonomy). On the other hand, the access control rule resulting from the logical and operator authorizes (resp. denies) access if both sub-rules authorize (resp. deny) the access.

Let us now suppose that $C l_{1} \neq C l_{2}$ and $C l_{1}^{\prime} \neq C l_{2}^{\prime}$. Then, the set of access control rules resulting from the combination of $\lambda_{1}$ and $\lambda_{2}$ depends not only on the operator for combining access control predicates, but also on the operators for combining classifications. Let $\delta_{1,2}$ (resp. $\delta_{1,2}^{\prime}$ ) be the operator defined for the combination of the $C l_{1}$ and $C l_{2}$ (resp. $C l_{1}^{\prime}$ and $C l_{2}^{\prime}$ ) classifications. Then, the set $\Lambda$ of access control rules takes one of the following forms, depending on the operators used for combining classifications:

- if $\delta_{1,2}=\chi$ or $\delta_{1,2}^{\prime}=\chi \nu$, then $\Lambda=\emptyset$ (the empty set), i.e. the combination is forbidden.

- if $\delta_{1,2} \in\{\cup, \cap\}$ and $\delta_{1,2}^{\prime} \in\{\cup, \cap\}$, then $\Lambda$ consists of a single access control rule: $\lambda=\left\{(s, o) \mid\left(s \in C l_{1} \delta_{1,2} C l_{2}\right) \wedge\left(o \in C l_{1}^{\prime} \delta_{1,2}^{\prime} C l_{2}^{\prime}\right) \wedge P_{1}(s, o)\right.$ op $\left.P_{2}(s, o) ; s \div>o\right\}$, where op $\in\{\vee, \wedge\}$.

- if $\delta_{1,2}=\uplus$, we only combine the access control predicates for subjects belonging to $C l_{1} \cap \mathrm{Cl}_{2}$, and we keep unchanged the rules for the other entities. In other words, $\Lambda$ consists of three separate access control rules:

$\lambda=\left\{(s, o) \mid\left(s \in\left(C l_{1}-C l_{2}\right)\right) \wedge\left(o \in\left(C l_{1}^{\prime} \delta_{1,2}^{\prime} C l_{2}^{\prime}\right)\right) \wedge P_{1}(s, o) ; s \div>o\right\}$, $\lambda^{\prime}=\left\{(s, o) \mid\left(s \in\left(C l_{1} \cap C l_{2}\right)\right) \wedge\left(o \in\left(C l_{1}^{\prime} \delta_{1,2}^{\prime} C l_{2}^{\prime}\right)\right) \wedge P_{1}(s, o)\right.$ op $\left.P_{2}(s, o) ; s \div>o\right\}$,

and

$\lambda^{\prime \prime}=\left\{(s, o) \mid\left(s \in\left(C l_{2}-C l_{1}\right)\right) \wedge\left(o \in\left(C l_{1}^{\prime} \delta_{1,2}^{\prime} C l_{2}^{\prime}\right)\right) \wedge P_{2}(s, o) ; s \div>o\right\}$, where op $\in\{\vee, \wedge\}$. If $\delta_{1,2}^{\prime}=\uplus$ or $\delta_{1,2}=\delta_{1,2}^{\prime}=\uplus$, the set $\Lambda$ of access control rules is equivalent to the one computed for $\delta_{1,2}=\uplus$.

Coupling the operators for combining classifications and the ones for combining access control predicates, allows either to restrict or to extend the access control rules of the subpolicies. In particular, using $\delta_{1,2}=\delta_{1,2}^{\prime}=\cap$ and the $\wedge$ operator for combining access control predicates, the access control rule $\lambda$ resulting from the composition of $\lambda_{1}$ and $\lambda_{2}$, verifies that: $(s, o) \in \lambda \Longleftrightarrow(s, o) \in \lambda_{1}$ and $(s, o) \in \lambda_{2}$, i.e. the access is authorized (resp. denied) if and only if it is authorized (resp. denied) by both $\lambda_{1}$ and $\lambda_{2}$ ( $\lambda$ is more specialized than $\lambda_{1}$ and $\lambda_{2}$ in the sense that it provides finest classifications). In this case, the composed policy keeps the principles of autonomy and secrecy of the sub-policies [14]: the combined policy is identical to the one obtained with policy interoperation.

When $\delta_{1,2}$ and $\delta_{1,2}^{\prime}$ belong to $\{\cup, \cap, \chi\}$, the combination of the $\lambda_{1}$ and $\lambda_{2}$ rules according to the $\wedge$ or $\vee$ operators can be expressed as:

$\mathrm{RR} \mathrm{n}^{\circ} 3112$ 
- $\quad \lambda_{1} \mathcal{R}_{\vee} \lambda_{2}=\left\{(s, o) \mid\left(s \in C l_{1,2}\right) \wedge\left(o \in C l_{1,2}^{\prime}\right) \wedge P_{1}(s, o) \vee P_{2}(s, o) ; s \div \circ o\right\}$, and

- $\lambda_{1} \mathcal{R}_{\wedge} \lambda_{2}=\left\{(s, o) \mid\left(s \in C l_{1,2}\right) \wedge\left(o \in C l_{1,2}^{\prime}\right) \wedge P_{1}(s, o) \wedge P_{2}(s, o) ; s \div>o\right\}$,

with $C l_{1,2}=C l_{1} \delta_{1,2} C l_{2}$ and $C l_{1,2}^{\prime}=C l_{1}^{\prime} \delta_{1,2}^{\prime} C l_{2}^{\prime}$. The reader should keep in mind that this notation is not appropriate for $\delta_{1,2}=\uplus$ or $\delta_{1,2}^{\prime}=\uplus$, because three separate rules are computed. However, we consider trivial to deduce formal expressions for them from those given above. Therefore, we use the above notation in the following.

Combination of conflicting rules. Let us now examine the combination of conflicting rules. Let $\lambda_{1}$ and $\lambda_{2}$ be two access control rules specifying respectively access authorization and access denial:

- $\lambda_{1}=\left\{(s, o) \mid\left(s \in C l_{1}\right) \wedge\left(o \in C l_{1}^{\prime}\right) \wedge P_{1}(s, o) ; s \sim>o\right\}$ and

- $\lambda_{2}=\left\{(s, o) \mid\left(s \in C l_{2}\right) \wedge\left(o \in C l_{2}^{\prime}\right) \wedge P_{2}(s, o) ; s \not>o o\right\}$.

Let $\delta_{1,2}$ (resp. $\delta_{1,2}^{\prime}$ ) be the operator defined for the combination of the classifications $C l_{1}$ and $C l_{2}$ (resp. $C l_{1}^{\prime}$ and $C l_{2}^{\prime}$ ). Let further $C l_{1,2}=C l_{1} \delta_{1,2} C l_{2}$ and $C l_{1,2}^{\prime}=C l_{1}^{\prime} \delta_{1,2}^{\prime} C l_{2}^{\prime}$. The $\mathcal{R}_{\wedge}^{+}$and $\mathcal{R}_{\wedge}^{-}$operators allow to combine two conflicting rules: $\lambda_{1} \mathcal{R}_{\wedge}^{+} \lambda_{2}$ and $\lambda_{1} \mathcal{R}_{\wedge}^{-} \lambda_{2}$. The result of the combination of $\lambda_{1}$ and $\lambda_{2}$ consists of three separate rules $\lambda, \lambda^{\prime}$ and $\lambda^{\prime \prime}$ defined by:

- $\lambda=\left\{(s, o) \mid\left(s \in C l_{1,2}\right) \wedge\left(o \in C l_{1,2}^{\prime}\right) \wedge\left(P_{1}(s, o) \wedge \neg P_{2}(s, o)\right) ; s \sim o\right\}$

- $\lambda^{\prime}=\left\{(s, o) \mid\left(s \in C l_{1,2}\right) \wedge\left(o \in C l_{1,2}^{\prime}\right) \wedge\left(\neg P_{1}(s, o) \wedge P_{2}(s, o)\right) ; s \not>o\right\}$

- $\lambda^{\prime \prime}=\left\{(s, o) \mid\left(s \in C l_{1,2}\right) \wedge\left(o \in C l_{1,2}^{\prime}\right) \wedge\left(P_{1}(s, o) \wedge P_{2}(s, o)\right) ; s\right.$ ac $\left.o\right\}$ with $($ ac $\equiv \sim>)$ for the operator $\mathcal{R}_{\wedge}^{+}$, and $($ac $\equiv \not>)$ for the operator $\mathcal{R}_{\wedge}^{-}$.

The $\lambda$ and $\lambda^{\prime}$ rules are coherent with both sub-rules. On the other hand, the $\lambda^{\prime \prime}$ rule is clearly inconsistent with either $\lambda_{1}$ or $\lambda_{2}$. The $\mathcal{R}_{\Lambda}^{+}$operator favors access authorization whereas the $\mathcal{R}_{\wedge}^{-}$operator favors access denial. Notice that the $\mathcal{R}_{\wedge}^{+}$and $\mathcal{R}_{\wedge}^{-}$operators are close to the notion of strong and weak authorizations introduced in database systems to support multiple access control policies [5].

As with the composition of non-conflicting rules, coupling the operators for combining classifications and the ones for combining access control rules allows either to restrict or to extend the sub-rules.

Finally, given the operators for combining classifications (i.e., the $\Delta$ set), and a set:

$$
\Psi=\left\{\alpha_{i, j} \in\left\{\mathcal{R}_{\wedge}, \mathcal{R}_{\vee}, \mathcal{R}_{\wedge}^{-}, \mathcal{R}_{\wedge}^{+}\right\}, 1, \ldots, n, j=1, \ldots, m\right\},
$$

we are able to combine two sets of access control rules $R_{1}=\left\{\lambda_{1}^{i}\right\}_{i=1}^{n}$ and $R_{2}=\left\{\lambda_{2}^{j}\right\}_{j=1}^{m}$ with respect to $\Delta$ and to $\Psi: R_{1} \mathcal{R}_{\Psi}^{\Delta} R_{2}$. Let $\mathcal{P}_{1}=\left(D o m_{1}, R_{1}\right)$ and $\mathcal{P}_{2}=\left(D o m_{2}, R_{2}\right)$ be two access control policies, and let further $\mathcal{P}=(D o m, R)$ be the access control policy resulting from the composition of $\mathcal{P}_{1}$ and $\mathcal{P}_{2}$ according to $\Delta$ and to $\Psi$ :

$$
\mathcal{P}=\mathcal{P}_{1} \mathcal{C}_{\Phi}^{\Delta} \mathcal{P}_{1}, \text { Dom }=\operatorname{Dom}_{1} \mathcal{D}_{\Delta} \text { Dom }_{2} \text { and } R=R_{1} \mathcal{R}_{\Phi}^{\Delta} R_{2}
$$

Let us remark that the proposed combination model applies recursively: a combined policy can be combined with another policy. 


\section{Example.}

We now illustrate the use of combination operators with the example of the file system given in $§ 3.1$. Given the component classifications $R E A D$ and $W R I T E$, the two sets of users $A$ and $C$, and the file classifications $B$ and $D$, we define the following access control policy $\mathcal{P}$ : the $(A-C)$ users are authorized to read files in $(B-D),(A \cap C)$ users can read and write files in $(B \cap D)$, and $(C-A)$ users are authorized to write files in $(D-B)$.

Using the $\uplus$ operator for combining classifications, we are able to differentiate components that only allow to read files $\left(R_{-} O N L Y=(R E A D-W R I T E)\right)$, components that only allow to write files $\left(W \_O N L Y=(W R I T E-R E A D)\right)$, and components that allow to read and to write files $\left(R_{-} a n d_{-} W=(R E A D \cap W R I T E)\right)$. The operator $\uplus$ further allows to differentiate the following sets of files: $B-D, B \cap D$ and $D-B$. Finally, using the operator $\mathcal{R}_{\wedge}$ for combining access control rules, we are able to get the specifications of $\mathcal{P}$ : $\lambda=\left\{(s, o) \mid\left(s \in R_{-} O N L Y\right) \wedge(o \in B-D) \wedge \operatorname{Owner}(s, A) ; s \sim>o\right\}, \lambda^{\prime}=\{(s, o) \mid(s \in$ $R_{-}$and_W $W \wedge(o \in(B \cap D)) \wedge($ Owner $(s, A) \wedge$ Owner $\left.(s, C)) ; s \sim>o\right\}$ and $\lambda^{\prime \prime}=\{(s, o) \mid(s \in$ $\left.W_{-} O N L Y\right) \wedge(o \in D-B) \wedge$ Owner $\left.(s, C) ; s \sim>o\right\}$.

\subsection{Complete and sound access control policy}

We have proposed a model for the specification of access control policies as well as a set of combination operators allowing to compose specifications of access control policies. Now, we define the notion of secure access control policy in the context of our model: the policy is secure if and only if it specification is complete and sound. Let us precisely define the completeness and soundness of policy specifications.

Definition 2 (Complete policy) An (access control) policy $\mathcal{P}$ is complete (from the standpoint of its specification) if and only if: (1) there exists an access control rule for every object and subject of $\mathcal{P}$ 's domain, and (2) in the case that $\mathcal{P}$ is a combined (access control) policy, its sub-policies are also complete.

In an open distributed architecture, an access control policy cannot be defined for the whole set of the system's entities. The above definition states that a complete access control policy ensures access control for any entity belonging to the policy's object domain, and for entity belonging to the policy's subject domain ${ }^{6}$.

Definition 3 (Sound policy) An (access control) policy $\mathcal{P}$ is sound (from the standpoint of its specification) if and only if: (1) given a subject $s$ and an object o belonging to the policy's domains, all the access control rules specifying whether $s$ can access o or not, have the same access control operator, and (2) in the case that $\mathcal{P}$ is a combined (access control) policy, its sub-policies are sound.

\footnotetext{
${ }^{6}$ In general, the completeness of a given policy can be guaranteed by specifying default access control rules.
}

$\mathrm{RR} \mathrm{n}^{\circ} 3112$ 
Given two sound sub-policies, if both specify only access authorization (or only denial) then for all combination operators, the combined policy is sound. On the opposite, when at least one of them specifies both authorized and denied accesses, the soundness of the combined policy may not be guaranteed. However, Gong et al. [14] have demonstrated that there exists a polynomial algorithm to check whether a given policy is sound. This result allows us to assert that, given two sub-policies and the combination operators between their domains and their access control rules, we are able to check whether the combined policy is sound or not.

When a combined access control policy is secure, we say that the sub-policies are nonconflicting. Finally, given two complete and sound access control policies, we have the following result which guarantees that given two access control policy, there exists a secure policy resulting from their combination.

Theorem 1 (Existence of secure combined access control policy) Let $\mathcal{P}_{1}$ and $\mathcal{P}_{2}$ be two secure access control policies. There exists a set $\Delta$ of operators for combining classifications and a set $\Psi$ of operators for combining access control rules such that, if $\mathcal{P}$ is the policy resulting from the combination of $\mathcal{P}_{1}$ and $\mathcal{P}_{2}$ according to the sets $\Delta$ and $\Psi$, then $\mathcal{P}$ is secure.

Proof: Let $\operatorname{Dom}_{1}=\left\{C l_{i}\right\}_{i=1}^{n}$ (resp. $\operatorname{Dom}_{2}=\left\{C l_{j}\right\}_{j=1}^{m}$ ) be $\mathcal{P}_{1}$ 's (resp. $\mathcal{P}_{2}$ 's) access control domains. Let $\Delta=\left\{\delta_{i, j}=\cap, i=1, \ldots, n, j=1, \ldots, m\right\}$. Let $R_{1}=\left\{\lambda_{1}^{i}\right\}_{i=1}^{n}$ (resp. $R_{2}=\left\{\lambda_{2}^{j}\right\}_{j=1}^{m}$ ) be $\mathcal{P}_{1}$ 's (resp. $\mathcal{P}_{2}$ 's) sets of access control rules. Let $\Psi=\left\{\alpha_{i, j} \in\right.$ $\left.\left\{\mathcal{C}_{\vee}, \mathcal{C}_{\wedge}^{+}, \mathcal{C}_{\wedge}^{-}\right\}, 1, \ldots, n, j=1, \ldots, m\right\}$ with

- $\alpha_{i, j}=\mathcal{C}_{\vee}$ if $\lambda_{1}^{i}$ and $\lambda_{2}^{j}$ are non-conflicting rules,

- $\alpha_{i, j}=\mathcal{C}_{\Lambda}^{+}$if $\lambda_{1}^{i}$ and $\lambda_{2}^{j}$ are conflicting rules and $\lambda_{1}^{i}$ is an access authorization rule,

- $\alpha_{i, j}=\mathcal{C}_{\wedge}^{-}$if $\lambda_{1}^{i}$ and $\lambda_{2}^{j}$ are conflicting rules and $\lambda_{1}^{i}$ is an access denial rule.

It is trivial to show that the policy $\mathcal{P}$ resulting from the composition of $\mathcal{P}_{1}$ and $\mathcal{P}_{2}$ according to the sets $\Delta$ and $\Psi$ is a complete and sound policy (i.e. $\mathcal{P}$ is secure).

\section{Example.}

Let us now address the completeness and soundness of policies specified in the file system example. The policies $\mathcal{P}_{1}$ and $\mathcal{P}_{2}$ are not complete. For instance, there are not access control rules concerning software components not belonging to subject's classifications and which require to access $B$ or $D$ files. If we specify default rules which deny all accesses between entities in $\mathcal{P}_{1}$ (resp. $\mathcal{P}_{2}$ ) security domain and the other entities, $\mathcal{P}_{1}$ (resp. $\mathcal{P}_{2}$ ) becomes complete. By giving priority to the access control rules over the default rules, the soundness of the policies $\mathcal{P}_{1}$ and $\mathcal{P}_{2}$ is also verified. Finally, by using the same default rules, the combined policy $\mathcal{P}$ is secure. 


\section{Extension for Information Flow policies}

This section deals with information flow policies. First, we extend the proposed model for specifying and combining access control policies to introduce the notion of information flow. Then, we address completeness and soundness of the resulting specifications, hence defining secure information flow policy according to our model.

\subsection{Information flow policy}

The previous model allows to describe access control policies but does not integrate any notion of information flow. Therefore, it does not meet the specification of information flow, as shown hereafter.

Let us consider two sets of users, $A$ and $C$, and two sets of files, $B$ and $D$, together with the following policy: (1) $A$ users are not authorized to read $B$ files, (2) $C$ users are authorized to read $B$ files, (3) $A$ users are authorized to read $D$ files and finally, (4) $C$ users are authorized to write $D$ files. With the help of a users belonging to $C$, a user of $A$ can access information related to $B$ files through $D$ files. This is contradictory to the policy's first rule. However, the policy is secure according to the previous model.

Since flow of information is a transitive operation, any flow policy must control all the transitive accesses. In order to deal with flow policies, we extend the previous model as follows: (1) the specifications of access control rules are enriched with the notion of information flow, and (2) rules that control the transitive information flow (by denying some information flows) are introduced.

\section{Input and output flows.}

For specifying the information flow that is associated to an access control rule, let us distinguish the access control rules for input flow (e.g. the read accesses) from the access control rules with output flow (e.g. the write accesses). We extend our definition of access control rules given in $\S 3.1$ by specifying the flow associated to the access operation with the flow operator. Given two entities $\epsilon_{1}$ and $\epsilon_{2}$, the expression $\epsilon_{1}$ flow $\epsilon_{2}$ specifies that there exists a flow from $\epsilon_{1}$ to $\epsilon_{2}$. We formally define rules for input flow and rules for output flow as follows:

Definition 4 (Rules for input and output flow) An access control rule for input flow (resp. output flow) takes the form $\varphi=\left\{(s, o) \mid(s \in C l) \wedge\left(o \in C l^{\prime}\right) \wedge P(s, o) ; s \sim>o ; s\right.$ flow $\left.o\right\}$ (resp. $\varphi=\left\{(s, o) \mid(s \in C l) \wedge\left(o \in C l^{\prime}\right) \wedge P(s, o) ; s \sim>o ; o\right.$ flow $\left.\left.s\right\}\right)$.

Note that we only specify the information flow for the authorized accesses.

\section{Information flow rules.}

The flow operator is transitive: let $\epsilon_{1}, \epsilon_{2}$ and $\epsilon_{3}$ be system entities, if $\epsilon_{1}$ flow $\epsilon_{2}$ and $\epsilon_{2}$ flow $\epsilon_{3}$ then $\epsilon_{1}$ flow $\epsilon_{3}$. In order to specify information flow policies, we specify the information flows which are denied, i.e. the information flow rules.

$\mathrm{RR} \mathrm{n}^{\circ} 3112$ 
Definition 5 (Information flow rule) An information flow rule is defined as $\phi=\left\{\left(\epsilon_{1}, \epsilon_{2}\right) \mid\left(\epsilon_{1} \in C l\right) \wedge\left(\epsilon_{2} \in C l^{\prime}\right) \wedge P\left(\epsilon_{1}, \epsilon_{2}\right) ; \neg\left(\epsilon_{1}\right.\right.$ flow $\left.\left.\epsilon_{2}\right)\right\}$.

The rule $\phi$ specifies that the information flow from $\mathrm{Cl}$ entities to $\mathrm{Cl}^{\prime}$ entities verifying the predicate $P$ is denied. Then, an information flow policy is defined as follows:

Definition 6 (Information flow policy) An information flow policy is an access control policy for which the access control rules are enriched according to the definition 4, and which defines a set of information flow rules.

\section{Example.}

The notion of multilevel security policy has been introduced in the late 60s by the U.S. Department of Defense for the protection of classified information stored in their computers [4]. Since then, various multilevel policies have been introduced to solve the problems in the industrial domains $[9,6]$.

In order to illustrate our model, we describe a simple multilevel policy called $\mathcal{F}_{S}$. We consider a distributed military system consisting of a set of software components (i.e. programs representing users), and a set of files that the components manipulate. Suppose that we have two security levels for both files and components: Secret and Unclassified ${ }^{7}$ such that Secret $>$ Unclassified (i.e. the Secret information is more sensible than the Unclassified information).

A software component can use a set of methods (e.g. read, write, execute, etc) to access files. In the following, we only deal with the read and write methods. Like in the example of $\S 3.1$, we define the $R E A D$ (resp. WRITE) classification to be the set of software components allowed to read (resp. write) files. We introduce the predicate $\left(\geq\left(\epsilon_{1}, \epsilon_{2}\right)\right)$ which, given two entities $\epsilon_{1}$ and $\epsilon_{2}$, holds if and only if the security level of $\epsilon_{1}$ dominates, i.e. is greater than or equal to the security level of $\epsilon_{2}$. Let us first define the following access control policy $\mathcal{P}_{S}$ :

- The subject domain consists of the READ and WRITE classifications, and the object domain consists of files belonging to Secret or Unclassified classifications.

- A component belonging to $R E A D$ is authorized to access a file if and only if its security level dominates the one of the file $\left(\lambda_{1}=\{(\right.$ s.o $) \mid(s \in R E A D) \wedge(o \in($ Secret $\cup$ Unclassified $)) \wedge \geq(s, o) ; s \sim>0\})$.

- A component belonging to WRITE is authorized to access an file if and only if the file security level dominates the one of the component $\left(\lambda_{2}=\{(s . o) \mid(s \in W R I T E) \wedge(o \in\right.$ Secret $\cup$ Unclassified $) \wedge(\geq(o, s)) ; s \sim>o\})$.

- All the accesses not authorized previously are denied through a default rule $(\{(s . o) \mid(s \in$ $R E A D \cup W R I T E) \wedge(o \in$ Secret $\cup$ Unclassified $) ; s \not>o\})$.

\footnotetext{
${ }^{7}$ The Secret and Unclassified terms are used for denoting both the security level, the classifications and the predicate.
} 
If we suppose that for any system entity $\epsilon, \epsilon$ has a unique security level, then the access control policy $\mathcal{P}_{S}$ is complete and sound.

We now define the information flow policy $\mathcal{F}_{S}$ as an extension of the access control policy $\mathcal{P}_{S}$. Given $\mathcal{P}_{S}$ 's access control rules, we define the information flow for each rule: $\lambda_{1}$ is a rule for input flow and $\lambda_{2}$ is a rule with output flow. Finally, we define the information flow rule of the policy $\mathcal{F}_{S}$ : Unclassified entities are not authorized to access Secret information: $\left(\phi_{S}=\left\{\left(\epsilon_{1}, \epsilon_{2}\right) \mid\left(\epsilon_{1} \in R E A D\right) \wedge\left(\epsilon_{2} \in\right.\right.\right.$ Secret $\cup$ Unclassified $) \wedge\left(\geq\left(\epsilon_{2}, \epsilon_{1}\right)\right) ; \neg\left(\epsilon_{1}\right.$ flow $\left.\left.\left.\epsilon_{2}\right)\right\}\right)$.

\subsection{Combined information flow policy}

Let us now address the combination of information flow policies. Given two information flow policies, we distinguish three steps for the combination of these policies: (1) the combination of these policies according to the operators for combining access control policies (see §3.2), (2) the combination of the flow operators in order to specify the information flow of each combined access control rule, and (3) the combination of information flow rules.

The first step allows to obtain the access control rules of the combined flow policy. During this step, the information flow sub-policies are viewed as access control policies, and the combination is carried out from the standpoint of combination of access control policies. The second step permits to integrate the flow operator within the combined access control policy obtained at the first step. Informally, the combination of two rules for input flow (resp. rules with output flow) is a rule for input flow (resp. rule with output flow). In the other case, the combined rule is a rule for both input and output flow.

Finally, we compute the combined information flow rules by composing the information flow rules of the sub-policies. In a way similar to the combination of non-conflicting rules, we identify two operators for the combination of information flow rules: the logical and and the logical or (see $\S 3.2 .2$ ). Let $\phi_{1}$ and $\phi_{2}$ be two information flow rules:

- $\phi_{1}=\left\{\left(\epsilon_{1}, \epsilon_{2}\right) \mid\left(\epsilon_{1} \in C l_{1}\right) \wedge\left(\epsilon_{2} \in C l_{1}^{\prime}\right) \wedge P_{1}\left(\epsilon_{1}, \epsilon_{2}\right) ; \neg\left(\epsilon_{1}\right.\right.$ flow $\left.\left.\epsilon_{2}\right)\right\}$ and

- $\phi_{2}=\left\{\left(\epsilon_{1}, \epsilon_{2}\right) \mid\left(\epsilon_{1} \in C l_{2}\right) \wedge\left(\epsilon_{2} \in C l_{2}^{\prime}\right) \wedge P_{2}\left(\epsilon_{1}, \epsilon_{2}\right) ; \neg\left(\epsilon_{1}\right.\right.$ flow $\left.\left.\epsilon_{2}\right)\right\}$.

Let $\delta_{1,2}$ (resp. $\delta_{1,2}^{\prime}$ ) be the operator defined for the combination of the classifications $C l_{1}$ and $C l_{2}$ (resp. $C l_{1}^{\prime}$ and $C l_{2}^{\prime}$ ). Then, the $\Phi$ set of information flow rules takes one of the following forms, depending on the operators used for combining classifications:

- if $\delta_{1,2}=\chi$ or $\delta_{1,2}^{\prime}=\not \nu$, then $\Phi=\emptyset$ (the empty set), i.e. the combination is forbidden.

- if $\delta_{1,2} \in\{\cup, \cap\}$ and $\delta_{1,2} \in\{\cup, \cap\}$, then $\Phi$ consists of a single information flow rule: $\phi=\left\{\left(\epsilon_{1}, \epsilon_{2}\right) \mid\left(\epsilon_{1} \in C l_{1} \delta_{1,2} C l_{2}\right) \wedge\left(\epsilon_{2} \in C l_{1}^{\prime} \delta_{1,2}^{\prime} C l_{2}^{\prime}\right) \wedge\right.$

$$
\left.P_{1}\left(\epsilon_{1}, \epsilon_{2}\right) \text { op } P_{2}\left(\epsilon_{1}, \epsilon_{2}\right) ; \neg\left(\epsilon_{1} \text { flow } \epsilon_{2}\right)\right\} \text {, }
$$

where op $\in\{\vee, \wedge\}$.

- if $\delta_{1,2}=\uplus$, we only combine the predicates for entities belonging to $C l_{1} \cap C l_{2}$, and we keep the same information flow rules for the rest of the entities (see §3.2.2). If $\delta_{1,2}^{\prime}=\uplus$ or $\delta_{1,2}=\delta_{1,2}^{\prime}=\uplus$, the $\Phi$ set of information flow rules is equivalent to the one computed for $\delta_{1,2}=\uplus$. 
Informally, the logical or operator allows to combine two information flow rules so as to generate a unique rule that provides the information flow control of the sub-policies. On the other hand, the information flow rule resulting from the logical and operator denies the information flow if and only if both sub-rules deny the flow.

\section{Example.}

Let us combine the information flow policy $\mathcal{F}_{S}$ introduced in $\S 4.1$ with another policy having different classifications. Suppose that we have two classifications in terms of military domains for both files and software components (e.g. users): Nuclear and Conventional classifications such as Nuclear > Conventional. Similarly, we define the information flow policy $\mathcal{F}_{D}$, except for the set of information flow rules that we define as an empty set.

By using the $\cap$ operator for combining classifications, and the $\mathcal{R}_{\wedge}$ operator for combining access control rules, we obtain the policy $\mathcal{F}$ depicted in figure 1 . For simplifying the figure, we do not distinguish components and files, and we do not show neither the denial accesses nor the implied transitive accesses. The integration of the flow operator in the combined access control rules are immediate according to the sub-policies' specifications (i.e. the read access produces input flow whereas the write access generates output flow). The information flow rule of the combined policy is the one of the policy $\mathcal{F}_{S}$ :

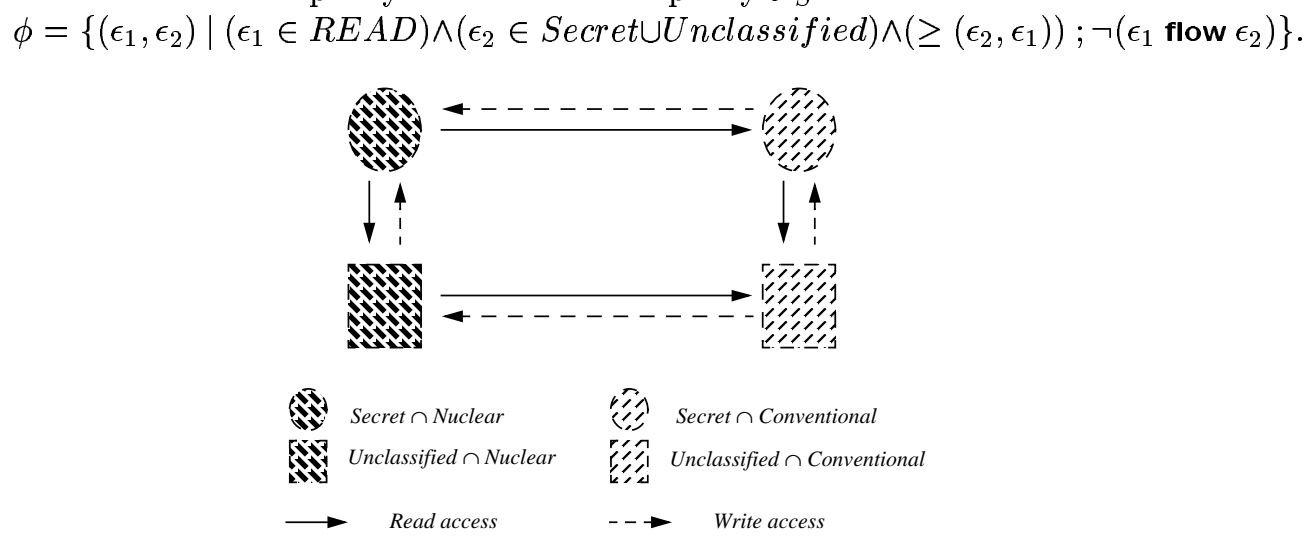

Figure 1: Combined multilevel policies

In order to obtain the policy introduced in $\S 2$, we further combine the policy $\mathcal{F}$ with the information flow policy authorizing the components belonging to Secret $\cap$ Conventional (resp. Unclassified $\cap$ Nuclear) to read (resp. write) entities belonging to Unclassified $\cap$ Nuclear (resp. Secret $\cap$ Conventional), and having no information flow rules.

\subsection{Complete and sound information flow policy}

Given the specifications of information flow policies in our model, we now focus on the notion of secure information flow policy by addressing the completeness and soundness properties. 
Definition 7 (Complete information flow policy) An information flow policy $\mathcal{F}$ is complete (from the standpoint of its specification) if and only if $\mathcal{F}$ is complete as an access control policy (see definition 2).

Given the specifications of an information flow policy, we can compute a tree representing the information flows between classifications. The edges of the graph are the different classifications whereas the arcs are given by the flow operator. We call information flow graph associated to the information flow policy the transitive closure of the previous graph. Given the information flow graph, we formally define the soundness property as follows:

Definition 8 (Sound information flow policy) An information flow policy $\mathcal{F}$ is sound (from the standpoint of its specification) if and only if: (1) $\mathcal{F}$ is sound as an access control policy, (2) the information flow graph of $\mathcal{F}$ does not invalidate the information flow rules of $\mathcal{F}$, and (3) in the case that $\mathcal{F}$ is a combined flow policy, its sub-policies are sound.

A secure information flow policy is a policy for which the specifications in our model are complete and sound. Like in $§ 3.3$, there exists a polynomial algorithm to check the soundness property, and given two information flow policies, there exists operators for combining classifications and rules such that the combined policy is secure.

The soundness property makes impossible the coexistence of two access control rules having the same information flow, but giving different access rights. This allows to prevent some covert channels. However, a policy being secure according to our model does not imply that the policy have no covert channels. For example, the write access is an access having output flow, but, when the file does not exist, the information flow induced by the write method is an input flow: after the operation, the user have information concerning the file's existence. Such covert channels do not invalidate the secure property.

Finally, a policy being secure according to our model implies that the policy's specifications are coherent with each other, and in the case of a combined policy, with the specification of the sub-policies according to the combination operators that are used.

\section{Example.}

Let us address the completeness and the soundness of the multilevel policies $\mathcal{F}_{S}$ and $\mathcal{F}_{D}$. Let us consider that the classifications in terms of security level or in terms of military domains are both complete classifications, i.e. any entity of the system has a (unique) security level and belongs to a (unique) military domains. Then the flow policies $\mathcal{F}_{S}$ and $\mathcal{F}_{D}$ becomes complete and sound access control policies. In the same way, the combined flow policy $\mathcal{F}$ is complete and sound as an access control policy.

The information graph of the policy $\mathcal{F}_{S}$ allows to check its soundness. Finally, the soundness of the policy $\mathcal{F}$ is trivial, that is $\mathcal{F}$ is a secure flow policy. 


\section{Conclusion}

In this paper, we have proposed a practical approach for reasoning about the coexistence of different security policies. Our approach relies on the specification of security policies and on the definition of operators for combining these specification. We have also addressed the completeness and the soundness of the (combined) security policy according to its specification.

Our model can be viewed as an implementation design of the Bell's model. We have introduced a set of combination operators enabling security officers to specify and to combine security policies in a controlled and secure manner. In addition, we assert that the dissociation between the combination domains and the combination rules permits to get a simplified and growing vision of security policies. Finally, the distinction between access operations and the generated information flows gives to our model a useful approach, information flow policies being an extension of access control policies.

We have illustrated our approach through two different examples of file systems in an distributed architecture. In particular, the multilevel security policy example enables us to demonstrate the strength for specifying security policies and the ease for combining security policies.

Introducing default operators for combining classification and rules in the security policy specifications allows to express the requirements of each policy concerning the operators to be used to combine this policy with another one. Thus, the combination of two security policies can be automatically computed, the soundness and the completeness of the combined policy allowing to verify that the two sub-policies are coherent with each other.

We are currently integrating our model in an configuration-based programming language similar to [21, 28], allowing to specify, at the application level, the security policy of each software component.

Concerning the extension of our model, we are interested with the dynamic management of classifications, in order to specify security policies with dynamic relabelling [12]. For example, the model presented in this paper does not allow to specify Chinese Wall policy [8]. The idea is to associate to each access control rule a function $F$ which is executed according to the access operator.

\section{References}

[1] M. Abadi and L. Lamport. Composing Specification. Technical Report 66, Digital Systems Research Center, Oct. 1990.

[2] M. Abrams, L. LaPadula, K. Eggers, and I. Olson. A Generalized Framework for Access Control: an Informal Description. In Proceedings of the 13th National Computer Security Conference, pages 134-143, Oct. 1990.

[3] D. E. Bell. Modeling the Multipolicy Machine. In Proceedings of the New Security Paradigm Workshop, pages 2-9, Aug. 1994. 
[4] D. E. Bell and L. J. LaPadula. Secure Computer Systems: Unified Exposition and Multics Interpretation. Technical Report MTR-2997 Rev. 1, MITRE Corporation, Bedford, Mass, 1976.

[5] E. Bertino, S. Jajodia, and P. Samarati. Supporting Multiple Access Control Policies in Database Systems. In Proceedings of the IEEE Symposium on Security and Privacy, pages 94-107, May 1996.

[6] K. J. Biba. Integrity consideration for secure computer systems. Technical Report MTR-2997, MITRE Corporation, Bedford, Mass, 1977.

[7] P. Bieber and F. Cuppens. A logical view of secure dependencies. Journal of Computer Security, 1(1):99-129, 1992.

[8] D. Brewer and M. Nash. The Chinese Wall Security Policy. In Proceedings of the IEEE Computer Society Symposium on Security and Privacy, pages 206-214, May 1989.

[9] D. Clark and D. Wilson. A Comparison of Commercial and Military Computer Security Policies. In I. C. Society, editor, Proceedings of the IEEE Symposium on Security and Privacy, 1987.

[10] R. Deng, S. Bhonsle, W. Wang, and A. Lazar. Integrating Security in CORBA Based Object Architectures. In Proceedings of the IEEE Symposium on Security and Privacy, pages 50-61, May 1995.

[11] Department of Defense Standard. Trusted Computer System Evaluation Criteria. Technical Report DoD 5200.28-STD, Dec. 1985.

[12] S. Foley, L. Gong, and X. Qian. A Security Model of Dynamic Labeling Providing a Tiered Approach to Verification. In Proceedings of the IEEE Symposium on Security and Privacy, pages 142-153, May 1996.

[13] J. Goguen and J. Meseguer. Security Policies and Security Models. In Proceedings of the IEEE Symposium on Security and Privacy, pages 11-20, May 1982.

[14] L. Gong and X. Qian. Computational issue in secure interoperation. IEEE Transactions on Software Engineering, 22(1):43-52, Jan. 1996.

[15] O. S. W. Group. White Paper on Security. TC Document 94.4.16, OMG, Apr. 1994. Available by ftp at ftp.omg.org:/pub/docs.

[16] N. Heintze and J. Tygar. A model for secure protocols and their compositions. IEEE Transactions on Software Engineering, 22(1):16-30, Jan. 1996.

[17] H. Hosmer. Metapolicies II. In Proceedings of the 15th National Computer Security Conference, pages 369-378, 1992.

[18] W. Kühnhauser and M. von Kopp Ostrowski. A Framework to Support Multiple Security Policies. In Proceedings of the 7th Canadian Computer Security Symposium, May 1995.

[19] W. E. Kühnhauser. A Paradigm for User-Defined Security Policies. In Proceedings of the Fourteenth Symposium on Reliable Distributed Systems, pages 135-144, 1995.

[20] C. E. Landwehr. Formal models for computer security. ACM Computing Surveys, 13(3):247278, Nov. 1981.

[21] J. Magee, N. Dulay, and J. Kramer. A Constructive Development for Parallel and Distributed Programs. In Proceedings of the International Workshop on Configurable Distributed Systems, 1994.

[22] J. McLean. The Algebra of Security. In Proceedings of the 1988 IEEE Computer Society Symposium on Security and Privacy, pages 2-7, Apr. 1988.

[23] J. McLean. Security Models and Information Flow. In Proceedings of the IEEE Symposium on Security and Privacy, pages 180-187, May 1990.

$\mathrm{RR} \mathrm{n}^{\circ} 3112$ 
[24] J. McLean. A general theory of composition for a class of possibilistic properties. IEEE Transactions on Software Engineering, 22(1):53-67, Jan. 1996.

[25] J. D. Moffett, M. D. Sloman, and K. Twidle. Specifying Discretionary Access Control Policy for Distributed Systems. Computer Communications, 13(9):571-580, Nov. 1990.

[26] National Computer Security Center. Trusted Network Interpretation of the TCSEC. Technical Report NCSC-TG-005, July 1987.

[27] OMG. The Common Object Request Broker: Architecture and Specification - Revision 2.0. Technical report, OMG Document, 1995.

[28] J. M. Purtilo. The Polylith software bus. ACM Transactions on Programming Languages and Systems, 16(1):151-174, 1994.

[29] D. Sutherland. A Model of Information. In Proceedings of the 9th National Computer Security Conference, pages 2-12, Sept. 1986.

[30] TINA-C. TINA Object Definition Language (TinA-OdL) Manual - Version 1.3. Technical Report TR_NM.002_1.3_95, TINA-C Document, 1995. 
Unit'e de recherche INRIA Lorraine, Technopôle de Nancy-Brabois, Campus scientifique, 615 rue du Jardin Botanique, BP 101, 54600 VILLERS LÈS NANCY

Unit'e de recherche INRIA Rennes, Irisa, Campus universitaire de Beaulieu, 35042 RENNES Cedex Unit'e de recherche INRIA Rhône-Alpes, 655, avenue de l'Europe, 38330 MONTBONNOT ST MARTIN Unit'e de recherche INRIA Rocquencourt, Domaine de Voluceau, Rocquencourt, BP 105, 78153 LE CHESNAY Cedex

Unit ${ }^{\prime} e$ de recherche INRIA Sophia-Antipolis, 2004 route des Lucioles, BP 93, 06902 SOPHIA-ANTIPOLIS Cedex

Éditeur

INRIA, Domaine de Voluceau, Rocquencourt, BP 105, 78153 LE CHESNAY Cedex (France)

http://www.inria.fr

ISSN 0249-6399 